\title{
Reflexive polytopes of higher index and the number 12
}

\author{
Alexander M. Kasprzyk \\ Department of Mathematics \\ Imperial College London \\ London, SW7 2AZ, UK \\ a.m.kasprzyk@imperial.ac.uk
}

\author{
Benjamin Nill \\ Department of Mathematics \\ Case Western Reserve University \\ Cleveland, OH, USA \\ benjamin.nill@case.edu
}

Submitted: May 19, 2012; Accepted: June 29, 2012; Published: July 19, 2012

Mathematics Subject Classifications: 52B20; 14M25, 14Q10

\begin{abstract}
We introduce reflexive polytopes of index $l$ as a natural generalisation of the notion of a reflexive polytope of index 1 . These $l$-reflexive polytopes also appear as dual pairs. In dimension two we show that they arise from reflexive polygons via a change of the underlying lattice. This allows us to efficiently classify all isomorphism classes of $l$-reflexive polygons up to index 200. As another application, we show that any reflexive polygon of arbitrary index satisfies the famous "number 12" property. This is a new, infinite class of lattice polygons possessing this property, and extends the previously known sixteen instances. The number 12 property also holds more generally for $l$-reflexive non-convex or self-intersecting polygonal loops. We conclude by discussing higher-dimensional examples and open questions.
\end{abstract}

Dedicated to the memory of Maximilian Kreuzer.

\section{Introduction and main results}

\subsection{Motivation}

Reflexive polytopes were first introduced by Batyrev in [4] in the context of Mirror Symmetry. In subsequent years they were intensively studied and classified as important examples of Fano varieties in toric geometry, and used in the construction of Calabi-Yau varieties (e.g., $[6,5,27,28,31,13,32])$. They are also intimately connected to commutative algebra and combinatorics via the study of Gorenstein polytopes $[7,2,12,3]$. Here we present a natural generalisation of reflexive polytopes and illustrate many combinatorial properties which parallel the reflexive case. 


\subsection{Notation}

We begin by recalling some basic definitions, and by fixing our notation.

Let $N \cong \mathbb{Z}^{n}$ be a lattice, and let $P \subset N_{\mathbb{Q}}:=N \otimes_{\mathbb{Z}} \mathbb{Q}$ be an $n$-dimensional lattice polytope; i.e., the set of vertices of $P$, denoted by $\mathcal{V}(P)$, is contained in the lattice $N$. We denote the interior of $P$ by $\operatorname{int}(P)$ and its boundary by $\partial P$. The set of facets (codimensionone faces) of $P$ is referred to by $\mathcal{F}(P)$. The volume of $P$ will always mean the normalised volume $\operatorname{Vol}(P)$ with respect to the ambient lattice $N$. Two lattice polytopes $P \subseteq N_{\mathbb{Q}}$ and $P^{\prime} \subseteq N_{\mathbb{Q}}^{\prime}$ are isomorphic (or unimodular equivalent) if there exists a lattice isomorphism $N \cong N^{\prime}$ mapping $\mathcal{V}(P)$ onto $\mathcal{V}\left(P^{\prime}\right)$.

A lattice point $x$ in $N \backslash\{\mathbf{0}\}$ is primitive if the line segment joining $x$ and $\mathbf{0}$ contains no other lattice points. We denote by $M$ the dual lattice $\operatorname{Hom}(N, \mathbb{Z})$ of $N$. Given a facet $F$ of $P$ we define its primitive outer normal to be the unique primitive lattice point $u_{F} \in M$ such that $F=\left\{x \in P \mid\left\langle u_{F}, x\right\rangle=l_{F}\right\}$ for some (uniquely determined) $l_{F} \in \mathbb{Z}_{>0}$. We call $l_{F}$ the local index of $F$; it is equal to the integral distance of $\mathbf{0}$ from the affine hyperplane spanned by $F$. The index (or Gorenstein index) of $P$ is defined as the least common multiple of the local indices of its facets.

\subsection{Reflexive polytopes of higher index}

Definition 1. A lattice polytope $P$ is called $l$-reflexive if, for some $l \in \mathbb{Z}_{>0}$, the following conditions hold:

i. $P$ contains the origin in its (strict) interior;

ii. The vertices of $P$ are primitive;

iii. For any facet $F$ of $P$ the local index $l_{F}$ equals $l$.

We also refer to $P$ as a reflexive polytope of index $l$.

The 1-reflexive polytopes are precisely the reflexive polytopes of [4]. Note that the requirement that the vertices are primitive prevents multiples of 1-reflexive polytopes from being $l$-reflexive.

\subsection{Duality}

Let $P \subseteq N_{\mathbb{Q}}$ be a full-dimensional lattice polytope. The dual polyhedron

$$
P^{*}:=\left\{y \in M_{\mathbb{Q}} \mid\langle y, x\rangle \leqslant 1\right\}
$$

is a (not necessarily lattice) polytope if and only if $\mathbf{0}$ lies in the interior of $P$. It is a well-known characterisation of reflexive polytopes that $P$ is reflexive if and only if $P^{*}$ is a lattice polytope (see [4]). In this case $P^{*}$ is also a reflexive polytope.

This characterisation has a natural reformulation for $l$-reflexive polytopes: 
Proposition 2. Let $P$ be a lattice polytope with primitive vertices, such that $P$ contains the origin in its interior. Then $P$ is l-reflexive if and only if $l P^{*}$ is a lattice polytope having only primitive vertices. In this case, $l P^{*}$ is also l-reflexive. This induces a natural duality for l-reflexive polytopes:

$$
P \longleftrightarrow l P^{*}
$$

Proof. Note that the vertices of $P^{*}$ are precisely the points $u_{F} / l_{F}$, for each facet $F$ of $P$. Analogously, the facets of $P^{*}$ are in one-to-one correspondence with the vertices of $P$.

If $P$ is $l$-reflexive then $l P^{*}=\left\{u_{F} \mid F \in \mathcal{F}(P)\right\}$, so any vertex of $l P^{*}$ is primitive. Moreover, any facet of $l P^{*}$ is given as $\left\{x \in l P^{*} \mid\langle v, x\rangle=l\right\}$ for some $v \in \mathcal{V}(P)$. Since the vertices of $P$ are primitive, it follows that any facet of $l P^{*}$ has local index $l$. Hence $l P^{*}$ is also $l$-reflexive.

Conversely, suppose that $l P^{*}$ is a lattice polytope having only primitive vertices. Then for any facet $F \in \mathcal{F}(P)$ we see that $l\left(u_{F} / l_{F}\right)$ is a primitive lattice point. Hence $l_{F}=l$ and $P$ is $l$-reflexive.

Finally, since

$$
l\left(l P^{*}\right)^{*}=l\left(\frac{1}{l} P\right)=P,
$$

the duality (1) follows by symmetry.

Notice that when $l=1$ we recover the usual duality of reflexive polytopes.

\subsection{Finiteness, examples, and classification}

A reflexive polytope (of index 1) contains only one interior lattice point, which is necessarily the origin. More generally, an $l$-reflexive polytope $P \subseteq N_{\mathbb{Q}}$ may contain several interior points, however it follows from the definition that

$$
\operatorname{int}(P / l) \cap N=\{\mathbf{0}\},
$$

or, equivalently, that $|\operatorname{int}(P) \cap l N|=1$. A result of Lagarias and Ziegler [29] implies that, for fixed dimension $n$ and index $l$, there are only finitely many isomorphism classes of $n$-dimensional $l$-reflexive polytopes (two polytopes are said to be isomorphic if there exists a unimodular transformation mapping one polytope to the other).

In dimension one there are no $l$-reflexive polytopes when $l>1$, and only one when $l=1$ : the line segment $[-1,1]$ corresponding to $\mathbb{P}^{1}$. In dimension two the $l$-reflexive polygons form a subset of the LDP-polygons studied in [26]. The reader is invited to try to find some $l$-reflexive polygons before proceeding. Whilst it is not too difficult to find all sixteen isomorphism classes of 1-reflexive polygons (draw any convex lattice polygon with no interior lattice points other than the origin), it is actually quite challenging to find examples of higher index. For instance, one quickly suspects that there is no reflexive polygon of index 2. Even more is true, as will be explained in Section 2.

Proposition 3. There is no l-reflexive polygon of even index. 
Up to isomorphism there is precisely one 3-reflexive polygon, which we denote by $P_{3}$ and is illustrated in Figure 1. This example generalises to a family of $l$-reflexive polygons, one for each odd index. Let $P_{l}$ be the polygon defined by the convex hull of $\{ \pm(0,1), \pm(l, 2), \pm(l, 1)\}$. This is a centrally-symmetric hexagon and, since $l$ is odd, $P_{l}$ is an $l$-reflexive polygon. To see this note that the vertices of $P_{l}^{*}$ are given by $\left\{ \pm\left(\frac{1}{l}, 0\right), \pm\left(\frac{2}{l},-1\right), \pm\left(\frac{1}{l},-1\right)\right\}$. Hence $P_{l}$ is actually self-dual in the sense that it is isomorphic to $l P_{l}^{*}$.

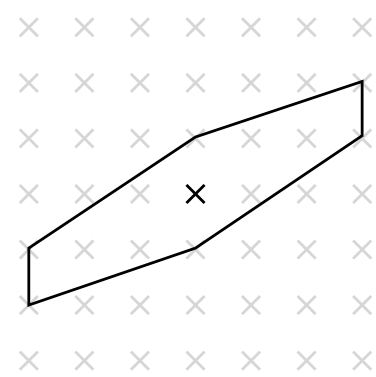

Figure 1: The unique 3-reflexive polygon $P_{3}$.

Notice that $P:=\operatorname{conv}\{ \pm(0,1), \pm(1,1), \pm(1,0)\}$ is, up to isomorphism, the unique 1reflexive polygon which is also a centrally-symmetric hexagon, and that $P_{l}=\varphi(P)$, where the map $\varphi$ is given by right multiplication with the matrix

$$
\left(\begin{array}{ll}
l & 1 \\
0 & 1
\end{array}\right)
$$

In Corollary 12 we will generalise this observation to any $l$-reflexive polygon. This gives a fast classification algorithm, which we implemented in MAgma (see Appendix A).

Theorem 4. For each positive odd integer $l$ let $n(l)$ be the number of isomorphism classes of l-reflexive polygons. Then, for $1 \leqslant l<60$ :

\begin{tabular}{|r|c|c|c|c|c|c|c|c|c|c|c|c|c|c|c|}
\hline$l$ & 1 & 3 & 5 & 7 & 9 & 11 & 13 & 15 & 17 & 19 & 21 & 23 & 25 & 27 & 29 \\
\hline$n(l)$ & 16 & 1 & 12 & 29 & 1 & 61 & 81 & 1 & 113 & 131 & 2 & 163 & 50 & 2 & 215 \\
\hline \hline$l$ & 31 & 33 & 35 & 37 & 39 & 41 & 43 & 45 & 47 & 49 & 51 & 53 & 55 & 57 & 59 \\
\hline$n(l)$ & 233 & 2 & 34 & 285 & 3 & 317 & 335 & 2 & 367 & 182 & 3 & 419 & 72 & 4 & 469 \\
\hline
\end{tabular}

A complete classification of the l-reflexive polygons up to index 200 is available online via the Graded Ring Database:

http://grdb.lboro.ac.uk/forms/toriclr2

Corollary 13 in Section 2 implies the following upper bound on the growth of $l$-reflexive polygons:

Corollary 5. There are at most $16(\phi(l)-1)$ isomorphism classes of l-reflexive polygons, where $\phi$ is Euler's totient function. 
It is an interesting phenomenon that lattice polygons with primitive vertices exhibit peculiar behaviours which have a number-theoretic flavour [15]. In particular, notice the astonishingly slow growth in the number of non-isomorphic $3 k$-reflexive polygons. Proposition 19 states that these are of a special form: they are self-dual hexagons. By inspecting the database, we also see that the number of isomorphism classes of self-dual $l$ reflexive polygons grows very slowly, although we do not know of a satisfactory explanation for this observation.

\subsection{The "number 12 "}

Recall that the famous "number 12 " property $[17,33,23,19]$ states that the sum of the number of boundary lattice points on a 1-reflexive polygon and the number of boundary lattice points on its dual always equals twelve. In Section 2 we extend this property to the class of reflexive polygons of higher index. While there are only sixteen reflexive polygons of index 1 up to isomorphism [33], there are infinitely many reflexive polygons of higher index.

Theorem 6. Let $P$ be a l-reflexive polygon. Then

$$
|\partial P \cap N|+\left|\partial\left(l P^{*}\right) \cap M\right|=12 .
$$

Note that $P$ and $l P^{*}$ have the same number of vertices.

Corollary 7. Any reflexive polygon of arbitrary index has at most nine boundary points and six vertices.

Corollary 8. Any (possibly singular) toric del Pezzo surface whose automorphism group acts transitively on the set of torus-invariant points has at most six torus-invariant points.

Our proof of Theorem 6 involves a purely combinatorial argument which reduces the statement to the "number 12" property for 1-reflexive polygons. This classical statement has a very elegant algebro-geometric proof (see [33]). We wonder whether there is also a direct argument arising from algebraic geometry in the case of $l$-reflexive polygons. We note that the toric surface associated with the fan spanned by the faces of an $l$-reflexive polygon is a log del Pezzo surface, i.e., a normal complex surface with ample $\mathbb{Q}$-Cartier anticanonical divisor $-K_{X}$ and at worst $\log$ terminal singularities (see $[1,26,30]$ ).

\subsection{Organisation of the paper}

In Section 2 we investigate $l$-reflexive polygons and consider a non-convex generalisation. We also prove the results given in Section 1. In Section 3 we describe higher-dimensional examples and state some open questions. 


\section{Dimension two}

\section{$2.1 \quad l$-reflexive loops}

There is a generalisation of reflexive polygons due to Poonen and Rodriguez-Villegas [33]. We give the analogous definition for higher index.

Definition 9. Let $x_{1}, \ldots, x_{t} \in N=\mathbb{Z}^{2}$ be non-zero lattice points, and define $x_{0}:=x_{t}$, $x_{t+1}:=x_{1}$. We say $\left\{x_{1}, \ldots, x_{t}\right\}$ is the set of boundary lattice points $\partial P \cap N$ of an l-reflexive loop $P$ of length $t$ if the following three conditions are satisfied for each $i=1, \ldots, t$ :

i. The lattice point $x_{i+1}-x_{i}$ is primitive;

ii. The determinant of the $2 \times 2$-matrix $A_{i}$ formed by $x_{i}, x_{i+1}$ equals $\pm l$;

iii. If $x_{i}$ is a vertex (i.e., $x_{i} \notin \operatorname{conv}\left\{x_{i-1}, x_{i+1}\right\}$ ) then it is primitive.

The length of $P$ is defined as $\sum_{i=1}^{t} \operatorname{det}\left(A_{i}\right) / l$. The set of facets of $P$ is naturally given as the set of line segments between successive vertices. Note that an $l$-reflexive loop may be a non-convex or self-intersecting polygonal loop (see Figure 2).

For $i=1, \ldots, t$, let $u_{i}$ be the primitive outer normal to the segment $\operatorname{conv}\left\{x_{i}, x_{i+1}\right\}$. Then

$$
\bigcup_{i=1}^{t} \operatorname{conv}\left\{u_{i}, u_{i+1}\right\} \cap M
$$

is the set of boundary lattice points of the dual l-reflexive loop $l P^{*}$ (see Figure 2). We leave it to the reader to check that this is well-defined, and that the duality of Proposition 2 generalises to this setting.

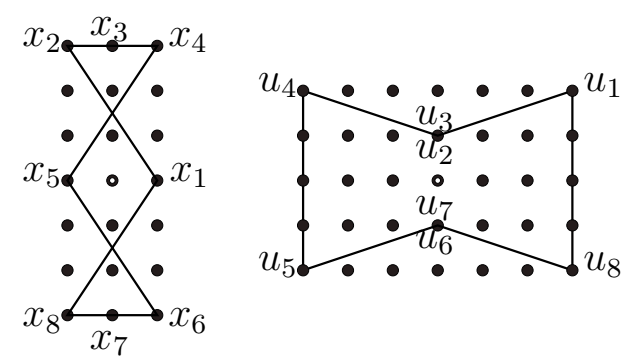

Figure 2: A 3-reflexive loop of length 0 and its dual of length 12.

\subsection{Change of lattice}

Throughout, let $P$ be an $l$-reflexive loop.

Definition 10. Let $\Lambda_{P}$ be the lattice generated by the boundary lattice points of $P$.

Here is our main technical result. 
Proposition 11. Let $P$ be an l-reflexive loop. Then

$$
\Lambda_{l P^{*}}=l \Lambda_{P}^{*}
$$

Moreover, $\Lambda_{P} \subseteq N$ and $l \Lambda_{P}^{*} \subseteq M$ are both lattices of index $l$.

Proof. Let us first show that if $x \in \partial P \cap N$ and $u_{F} \in \mathcal{V}\left(l P^{*}\right)$ then $\left\langle u_{F}, x\right\rangle \in l \mathbb{Z}$. We prove this by inductively showing that if $F_{1}, \ldots, F_{s}$ is a successive sequence of facets of $P$ (in either clockwise or counter-clockwise order) such that $F_{i}$ is adjacent to $F_{i-1}$ (for $i=2, \ldots, s)$, and if $F_{s+1}$ is the other facet adjacent to $F_{s}$, then $\left\langle u_{F_{1}}, x\right\rangle \in l \mathbb{Z}$ for any point $x \in F_{s+1} \cap N$.

By definition we only have to consider $s>1$. We may assume by a unimodular transformation that $u_{F_{1}}=(0,1)$. Let $u_{F_{s+1}}=(a, b), x=(c, d) \in F_{s+1} \cap N$, and $v=$ $(k, l) \in \mathcal{V}\left(F_{1} \cap F_{2}\right)$. Applying the induction hypothesis to $F_{s+1}, F_{s}, \ldots, F_{2}$, yields that $l$ divides $\left\langle u_{F_{s+1}}, v\right\rangle=a k+b l$. Therefore, $l$ divides $a k$. Since $v$ is primitive, $\operatorname{gcd}\{k, l\}=1$, hence, $l$ divides $a$. We know that $\left\langle u_{F_{s+1}}, x\right\rangle=a c+b d=l$. Therefore, $l$ divides $b d$. Since, $u_{F_{s+1}}$ is primitive, $\operatorname{gcd}\{a, b\}=1$, so $\operatorname{gcd}\{l, b\}=1$, hence $l$ divides $d=\left\langle u_{F_{1}}, x\right\rangle$ as desired.

By symmetry we may assume $x \in \operatorname{int}(F) \cap N$ and $y \in \operatorname{int}(G) \cap M$, where $F \in \mathcal{F}(P)$ and $G \in \mathcal{F}\left(l P^{*}\right)$. Let $u_{F} \in \mathcal{V}\left(l P^{*}\right)$ and $v_{G} \in \mathcal{V}(P)$ be the corresponding primitive outer normals. We may again assume that $v_{G}=(0,1)$. Let $u_{F}=(a, b), x=(c, d), y=(k, l)$. As we have seen, $l$ divides $\left\langle v_{G}, u_{F}\right\rangle=b$. On the other hand, $l=\left\langle u_{F}, x\right\rangle=a c+b d$, so $l$ divides $a c$. Since $\operatorname{gcd}\{a, b\}=1$, so $\operatorname{gcd}\{a, l\}=1$, hence $l$ divides $c$. Therefore, $l$ divides $c k+d l=\langle x, y\rangle$.

This shows $\Lambda_{l P^{*}} \subseteq l \Lambda_{P}^{*}$. To show the converse direction, let $F$ be a facet of $P$. We denote by $\Lambda_{F}$ the lattice generated by the lattice points in $F$. We may assume that $u_{F}=(0,1)$ and hence $\Lambda_{F}$ is generated by $(1,0),(0, l)$. For any boundary lattice point $x$ in $P$, we have shown that $l$ divides $\left\langle u_{F}, x\right\rangle$, hence $x \in \Lambda_{F}$. This proves $\Lambda_{P}=\Lambda_{F}$. In particular, $\Lambda_{P} \subseteq N$ has index $l$. By symmetry, $\Lambda_{l P^{*}} \subseteq M$ also has index $l$, and we have that $\Lambda_{P}^{*}$ is generated by $(1,0)$ and $(0,1 / l)$. Therefore $l \Lambda_{P}^{*} \subseteq M$ is also a lattice of index $l$. Since $\Lambda_{l P^{*}} \subseteq l \Lambda_{P}^{*}$ are sublattices of $M$ of the same index, they are equal.

\subsection{Applications and proofs of results in Section 1}

We describe several corollaries to Proposition 11.

Corollary 12. Let $P$ be an l-reflexive loop. Then $P$ is a 1-reflexive loop with respect to the lattice $\Lambda_{P}$, which we call the 1-reflexive loop associated to $P$. Moreover, its dual 1-reflexive loop is isomorphic to the 1-reflexive loop associated to $l P^{*}$.

Proof. By Proposition 11, $\Lambda_{l P^{*}} / l=\Lambda_{P}^{*}$. Since any vertex of $P^{*}$ is of the form $u / l$ for some (primitive) vertex $u$ of $l P^{*}$, the vertices of $P^{*}$ lie in $\Lambda_{P}^{*}$. They are necessarily primitive, since for any vertex $w \in \mathcal{V}\left(P^{*}\right)$ there is a vertex $v \in \mathcal{V}(P)$ such that $\langle w, v\rangle=1$. Therefore by Proposition $2 P^{*}$ is a 1 -reflexive loop, say $Q^{*}$, with respect to $\Lambda_{P}^{*}$. Moreover, via multiplication by $l, Q^{*}$ is isomorphic to $l P^{*}$ with respect to the lattice $\Lambda_{l P^{*}}$. 
Using this we can derive an efficient classification algorithm for $l$-reflexive polygons analogous to an approach by Conrads [14], see Appendix A.

Corollary 13. Let $\mathcal{R}$ be a set of representatives of all isomorphism classes of 1-reflexive polygons (respectively, loops). We may choose any $Q \in \mathcal{R}$ such that $(0,1) \in N$ is a vertex of $Q$ and $(0,1) \in M$ is a vertex of $Q^{*}$. If $P$ is an l-reflexive polygon (respectively, loop) of index $l \geqslant 2$, then there exists $Q \in \mathcal{R}$ such that $P$ is isomorphic to the image of $Q$ under the map

$$
\left(\begin{array}{ll}
l & i \\
0 & 1
\end{array}\right)
$$

for $0<i<l$ coprime to $l$.

Proof. By Proposition 11 there is an isomorphism $\mathbb{Z}^{2} \rightarrow \Lambda_{P} \subseteq \mathbb{Z}^{2}$ given by rightmultiplying an integer $2 \times 2$-matrix $H^{\prime}$ of determinant $l$. Corollary 12 yields that $P=Q^{\prime} H^{\prime}$ for some 1-reflexive polygon (equiv. loop) $Q^{\prime}$. Thus, by our assumption, there exists $Q \in \mathcal{R}$ and a unimodular $2 \times 2$-matrix $U^{\prime}$ such that $Q^{\prime}=Q U^{\prime}$, hence, $U^{\prime} H^{\prime}$ maps $Q$ onto $P$. The Hermite normal form theorem yields that there exists a unimodular $2 \times 2$-matrix $U$ such that $H:=U^{\prime} H^{\prime} U$ is in upper triangular Hermite normal form

$$
\left(\begin{array}{cc}
d & i \\
0 & l / d
\end{array}\right)
$$

for $d$ a divisor of $l$, and $0 \leqslant i<d$. Therefore $Q$ maps via $H$ onto the image $P^{\prime}$ of $P$ under $U$. Since $(0,1)$ is a vertex of $Q$, the row vector $(0, l / d)$ is a vertex of the $l$-reflexive polygon (equiv. loop) $P^{\prime}$ and hence is primitive. Therefore $l / d=1$.

Let us consider the dual picture. One checks that $l\left(P^{\prime}\right)^{*}$ is equal to the image of $Q^{*}$ under the matrix

$$
M:=l\left(H^{T}\right)^{-1}=\left(\begin{array}{cc}
1 & 0 \\
-i & l
\end{array}\right)
$$

Define $g:=\operatorname{gcd}\{l, i\} \neq 0$. There exist unique integers $j, k$ with $0 \leqslant j<l / g$ such that

$$
-j i+k l=g
$$

Therefore, we can define an integer matrix $J$ with $\operatorname{det}(J)=1$ by setting

$$
J:=\left(\begin{array}{cc}
\frac{l}{g} & j \\
\frac{i}{g} & k
\end{array}\right) .
$$

Hence

$$
M \cdot J=\left(\begin{array}{cc}
\frac{l}{g} & j \\
0 & g
\end{array}\right)=: K
$$

is in Hermite normal form. Therefore $l\left(P^{\prime}\right)^{*}$ is isomorphic to the image of $Q^{*}$ under the matrix $K$. As above, our assumption that $(0,1)$ is a vertex of $Q^{*}$ implies that $g=1$, as desired. 
Remark 14. Notice that if

$$
H:=\left(\begin{array}{ll}
l & i \\
0 & 1
\end{array}\right) \quad \text { and } \quad K:=\left(\begin{array}{ll}
l & j \\
0 & 1
\end{array}\right)
$$

are the matrices in Corollary 13 yielding $P$ and $l P^{*}$ then, by equation $(2), i j \equiv-1(\bmod l)$.

Since there are only sixteen non-isomorphic reflexive polygons (see, e.g., [33]), this gives a very rapid algorithm for classifying $l$-reflexive polygons. It also explains why Corollary 5 holds.

Let us prove Theorem 6 in the more general setting of $l$-reflexive loops. Note that $l$-reflexive loops have a well-defined winding number $w(P) \in \mathbb{Z}$ (see [33] in the case of 1-reflexive loops; then apply Corollary 12).

Corollary 15. Let $P$ be an l-reflexive loop. Then the sum of the length of $P$ and the length of $l P^{*}$ equals $12 w(P)$.

Proof. Let $Q$ be the associated 1-reflexive loop with respect to the lattice $L:=\Lambda_{P}$. By [33] we know that the desired statement holds for the pair $Q, Q^{*}$. Let $b_{N}(P)$ denote the number of boundary lattice points of an $l$-reflexive loop $P$. By definition $b_{L}(Q)=b_{N}(P)$, and by Proposition 11 and Corollary 12, $b_{L^{*}}\left(Q^{*}\right)=b_{l L^{*}}\left(l Q^{*}\right)=b_{\Lambda_{l P^{*}}}\left(l P^{*}\right)=b_{M}\left(l P^{*}\right)$.

It would be interesting to prove Corollary 15 directly by generalising the proof for 1-reflexive loops as given in [33].

Finally, we prove Proposition 3, which states that there are no $l$-reflexive polygons of even index $l$. Experimental evidence suggests that this statement should also hold for $l$-reflexive loops, however we do not yet know how to generalise it to the non-convex setting.

Proof of Proposition 3. Assume $l$ is even. Let $F$ be a facet of $P$. We may assume that its vertices are given as $(a, l)$ and $(b, l)$. Since the vertices of $P$ are primitive, $a$ and $b$ are odd. Therefore, the midpoint $\frac{(a, l)+(b, l)}{2}$ of the facet $F$ is a lattice point.

By symmetry, this shows that any facet of $P$ and of $l P^{*}$ contains an interior lattice point. By Corollary 12, this property also holds for $Q$ and $Q^{*}$, where $Q$ is the associated 1-reflexive polygon. However, by inspecting the list of sixteen isomorphism classes of 1-reflexive polygons we see that this is not possible.

\subsection{Roots of Ehrhart polynomials of l-reflexive polygons}

In this section we shall discuss roots of Ehrhart polynomials and generalise some results concerning reflexive polygons.

By Corollary 12 any statement about boundary lattice points or vertices of a 1-reflexive polygon also holds for $l$-reflexive polygons. For instance, it is clear that $l$-reflexive polygons have at most 6 vertices. In any dimension, the normalised volume of an $l$-reflexive polytope is related to the boundary volume via $\operatorname{Vol}(P)=l \operatorname{Vol}(\partial P)$. In the two dimensional case, 
the normalised volume can be easily computed as $\operatorname{Vol}(P)=l b$, where $b$ is the number of boundary lattice points of $P$ (and, in particular, $3 \leqslant b \leqslant 9$ ).

Let $L_{P}(m):=|m P \cap N|$ denote the number of lattice points in $P$ dilated by a factor of $m \in \mathbb{Z}_{\geqslant 0}$. This is known to be a polynomial of degree $d:=\operatorname{dim} P$, called the Ehrhart polynomial. The roots of $L_{P}$ (regarded as a polynomial over $\mathbb{C}$ ) have been the subject of much study, e.g., $[8,22]$. In particular, it is known that if all roots $z \in \mathbb{C}$ of $L_{P}(m)$ satisfy $\operatorname{Re}(z)=-1 / 2$, then $P$ is a reflexive polytope [11, Proposition 1.8]. In dimension two we can generalise this result:

Proposition 16. Let $P$ be an LDP-polygon of index $l$ such that for all roots $z \in \mathbb{C}$ of $L_{P}(m), \operatorname{Re}(z)=-1 /(2 l)$. Then $P$ is an l-reflexive polygon.

Proof. Let $-1 /(2 l) \pm \alpha i$ be the two roots of $L_{P}(m)$. Then

$$
\begin{aligned}
L_{P}(m) & =\beta\left(m+\frac{1}{2 l}+\alpha i\right)\left(m+\frac{1}{2 l}-\alpha i\right) \\
& =\beta m^{2}+\frac{\beta}{l} m+\beta\left(\frac{1}{4 l^{2}}+\alpha^{2}\right),
\end{aligned}
$$

hence $\beta=(1 / 2) \operatorname{Vol}(P), \beta\left(1 /\left(4 l^{2}\right)+\alpha^{2}\right)=1$, and

$$
\operatorname{Vol}(P)=l \operatorname{Vol}(\partial P) .
$$

Let $F \in \mathcal{F}(P)$ be an edge, and let $l_{F}$ be the corresponding local index. The above result tells us that

$$
\sum_{F \in \mathcal{F}(P)}\left(l-l_{F}\right) \operatorname{Vol}(F)=0 .
$$

But $l_{F} \leqslant l$ for all $F \in \mathcal{F}(P)$, hence $l_{F}=l$ and so $P$ is $l$-reflexive.

While the converse is not true even for 1-reflexive polygons (see [11, 20]) we can give the following more precise statement:

Proposition 17. Let $P$ be an l-reflexive polygon not isomorphic to the convex hull of $\{(-1,-1),(-1,2),(2,-1)\}$ (see Figure 3). If $z \in \mathbb{C}$ is a root of $L_{P}(m)$, then $\operatorname{Re}(z)=$ $-1 /(2 l)$.

Proof. It follows from Pick's theorem (e.g., [10, Thm.2.9]) that

$$
L_{P}(m)=\frac{l b}{2} m^{2}+\frac{b}{2} m+1
$$

Let $z \in \mathbb{C}$ be a root of $L_{P}$. We get

$$
z=-\frac{1}{2 l} \pm \frac{\sqrt{b^{2}-8 l b}}{2 l b} .
$$

Since $b^{2}-8 l b \leqslant 0$ for all $3 \leqslant b \leqslant 9$ and $l \geqslant 1$ with the exception of $b=9, l=1$, the result follows. 


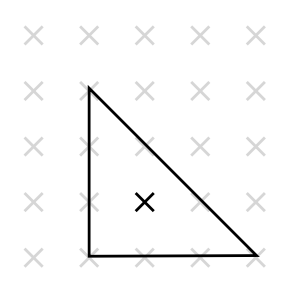

Figure 3: The unique exception to Proposition 17.

Remark 18. Using Pick's theorem it is also straightforward to compute the generating function $\operatorname{Ehr}_{P}$ of the Ehrhart polynomial $L_{P}$ :

$$
\operatorname{Ehr}_{P}(t):=\sum_{m \geqslant 0} L_{P}(m) t^{m}=\frac{h_{P}^{*}(t)}{(1-t)^{3}}
$$

where

$$
h_{P}^{*}(t)=1+(|P \cap N|-3) t+i t^{2}=1+\left(\frac{l+1}{2} b-2\right) t+\left(\frac{l-1}{2} b+1\right) t^{2} .
$$

The coefficients of the numerator $h_{P}^{*}$ are palindromic if and only if $l=1$.

In general, for any $l$-reflexive polytope $P$ of dimension $n$, the (rational) polytope $P / l$ has Ehrhart generating function

$$
\operatorname{Ehr}_{P / l}(t)=\frac{h_{P / l}^{*}(t)}{\left(1-t^{l}\right)^{n+1}},
$$

where $h_{P / l}^{*}$ is a polynomial of degree $l(n+1)-1$ with palindromic coefficients. This holds by [16], since the dual polytope $l P^{*}$ is a lattice polytope by Proposition 2 . When $l=1$ we recover the well-known result by Hibi [21].

\section{$2.53 k$-reflexive polygons}

As observed in the introduction, there are only very few $l$-reflexive polygons for $l$ divisible by 3 . Here, we give a partial explanation of this surprising phenomenon.

Proposition 19. Let $P$ be a $3 k$-reflexive polygon, where $k$ is an odd positive integer. Then $P$ can be obtained from the 1-reflexive hexagon $Q:=\operatorname{conv}\{ \pm(0,1), \pm(1,1), \pm(1,0)\}$ (in the sense of Corollary 13). Furthermore, $P \cong 3 k P^{*}$.

Proof. Let $P:=Q H$ be a $3 k$-reflexive polygon, where

$$
H:=\left(\begin{array}{cc}
3 k & i \\
0 & 1
\end{array}\right), \quad \operatorname{gcd}\{3 k, i\}=1 .
$$


On the dual side, by Remark 14 we have that $3 k P^{*} \cong Q^{*} K$ where

$$
K:=\left(\begin{array}{cc}
3 k & j \\
0 & 1
\end{array}\right), \quad \operatorname{gcd}\{3 k, j\}=1, \quad i j \equiv-1(\bmod 3 k) .
$$

Hence if $i \equiv 1(\bmod 3)$ then $j \equiv 2(\bmod 3)$, and if $i \equiv 2(\bmod 3)$ then $j \equiv 1(\bmod 3)$. We shall consider the sixteen possible choices for $Q$, and exclude all but the self-dual hexagon.

i. Suppose (after possible change of basis) that the vertices of $Q$ include the points $(0,1),(2,1)$, and $(-1,-1)$ (i.e., $Q$ contains the triangle associated with $\mathbb{P}(1,1,2))$. Then $(6 k, 2 i+1)$ and $(-3 k,-i-1)$ are vertices of $P$. But one of these points must be divisible by 3 , and hence is not primitive. This allows us to exclude the first six polygons in Figure 4(a), along with their duals. Up to isomorphism, this excludes all eight polygons depicted in Figure 4(a).

ii. Now suppose that $\mathcal{V}(Q)$ contains $(0,1),(1,1)$, and $(-1,-2)$ (i.e., the triangle associated with $\left.\mathbb{P}^{2}\right)$. Then $(3 k, i+1)$ and $(-3 k,-i-2)$ are vertices of $P$. Once again we see that these cannot both be primitive, excluding the first two polygons in Figure 4(b) and their duals. This excludes the four polygons shown in Figure 4(b).

iii. Let $Q:=\operatorname{conv}\{(0,1),(3,1),(-1,-1)\}$ be the triangle associated with $\mathbb{P}(1,2,3)$. Then $(-3 k,-i-1)$ is a vertex of $P$, forcing $i \equiv 1(\bmod 3)$. The dual $Q^{*}$ has vertices $\{(-2,1),(0,1),(1,-2)\}$; in particular $(3 k, j-2)$ is a vertex of $Q^{*} K$, giving $j \equiv 1(\bmod 3)$. This is a contradiction.

iv. Consider $Q:=\operatorname{conv}\{ \pm(0,1), \pm(1,1)\}$ (the polygon associated with $\mathbb{P}^{1} \times \mathbb{P}^{1}$ ). We see that $Q H$ has vertex $(3 k, i+1)$, giving $i \equiv 1(\bmod 3)$. On the dual side, $Q^{*}$ has vertices $\{ \pm(0,1), \pm(-2,1)\}$. This gives $(-6 k,-2 j+1) \in \mathcal{V}\left(Q^{*} K\right)$, again forcing $j \equiv 1(\bmod 3)$. This excludes the final two cases.

The only remaining possibility is that $Q$ is the self-dual hexagon with vertices $\{ \pm(0,1)$, $\pm(1,1), \pm(1,0)\}$. We show by direct calculation that $P=Q H$ is also self-dual. The vertices of $P$ are given by $\{ \pm(0,1), \pm(l, i+1), \pm(l, i)\}$, and the vertices of $l P^{*}=Q^{*} l\left(H^{t}\right)^{-1}$ are $\{ \pm(i,-l), \pm(i+1,-l), \pm(1,0)\}$. These are clearly isomorphic.

Note that any $i$ such that $\operatorname{gcd}\{3 k, i\}=\operatorname{gcd}\{3 k, i+1\}=1$ will give a $3 k$-reflexive hexagon, however these need not be distinct. With more care, it is possible to give precise

number-theoretic conditions for this to happen. This allows a very rapid enumeration of the possible $i$ that give non-isomorphic $3 k$-reflexive hexagons.

\section{$3 \quad$ Examples and open questions in higher dimensions}

\subsection{Motivational questions}

Motivated by the positive results in dimension two, there are many natural questions one may ask about l-reflexive polytopes in higher dimensions. Which of the results in dimension two extend to higher dimensions? What other properties of 1-reflexive polytopes 

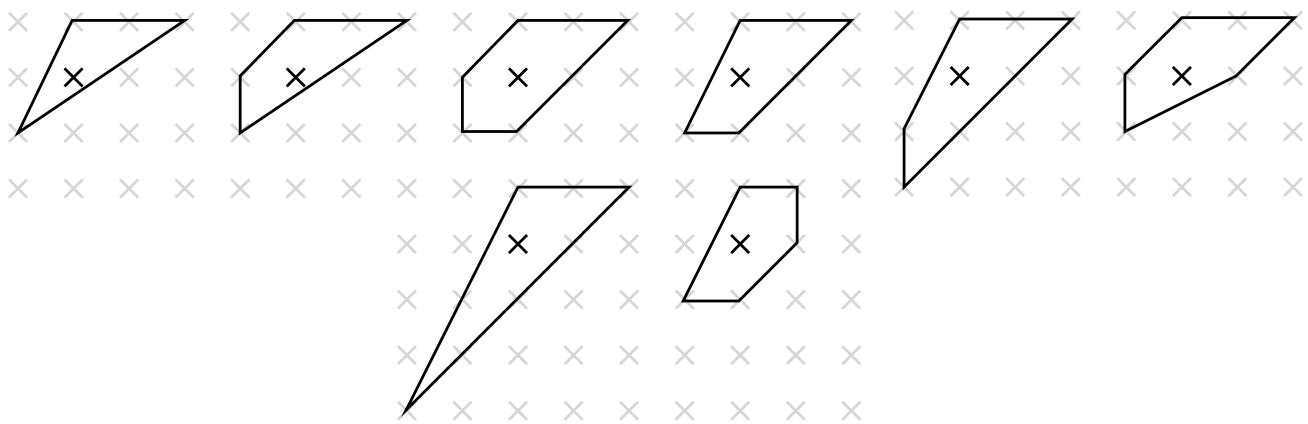

(a) Case (i)
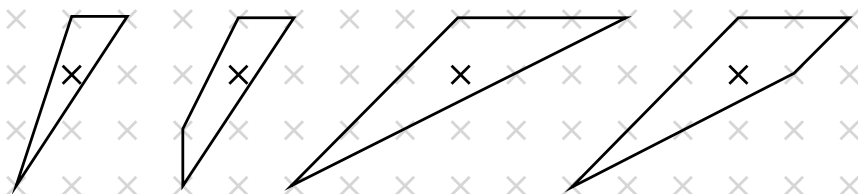

(b) Case (ii)

Figure 4: The polygons excluded in cases (i) and (ii) of the proof of Proposition 19.

can be generalised to higher index? Do the corresponding hypersurfaces have interesting properties - at least, if the we assume that the ambient space has mild (say, isolated) singularities? What about possible relations to Mirror Symmetry and Calabi-Yau varieties, which spurred the initial interest in 1-reflexive polytopes [4]? We remark that Gorenstein polytopes (lattice polytopes where some $r^{\text {th }}$-multiple is reflexive) may be regarded as being " $\frac{1}{r}$-reflexive"; they also satisfy a beautiful duality and are related to the construction of mirror-symmetric Calabi-Yau complete intersections [7, 3]. Can we say something similar about " $\frac{l}{r}$-reflexive polytopes"?

As we will illustrate below in the three-dimensional situation, we cannot expect direct generalisations of most results given in this paper. However, we are nevertheless convinced that there are many interesting results about (possibly subclasses of) $l$-reflexive polytopes in higher dimensions, and that their study is worthwhile.

\subsection{Any index is possible in dimension three}

In dimension two, $l$-reflexive polygons only exist for odd $l$. This is not true in dimension three. Let $P$ be the tetrahedron with vertices $\{(-l,-1,0),(l, 0,-1),(0,1,0),(0,0,1)\}$. Then $P^{*}$ is the convex hull of $\{(-2 / l,-1,-1),(2 / l,-1,-1),(-2 / l, 3,-1),(2 / l,-1,3)\}$. Therefore $P$ is $l$-reflexive if $l$ is odd, and $l / 2$-reflexive if $l$ is even. In particular, there exist three-dimensional $l$-reflexive polytopes for any index.

\subsection{Two examples in dimension three}

The main results in Section 2 fail to hold in dimension three. Perhaps the simplest counterexample is the tetrahedron $P$ with vertices $\{(1,0,0),(3,4,0),(5,0,8),(-9,-4,-8)\}$. 
This is a 2-reflexive polytope with $\Lambda_{P}=N$, hence neither Proposition 11 nor Corollary 12 generalise to higher dimensions.

The toric variety corresponding to $P$ is a fake weighted projective space, $\mathbb{P}^{3} /(\mathbb{Z} / 4 \times$ $\mathbb{Z} / 8)$. By definition we have that $P$ restricted to $\Lambda_{\mathcal{V}(P)}$ is the 1-reflexive simplex associated with $\mathbb{P}^{3}$. The dual polytope $2 P^{*}$ is also 2-reflexive. In this case the corresponding toric variety is $\mathbb{P}^{3} /(\mathbb{Z} / 4)$, which has canonical singularities ${ }^{1}$. Interestingly, $2 P^{*}$ has only the origin as an interior lattice point, whilst its index is 2 . Such behaviour is not possible in dimension two. Notice that $P$ is 1-reflexive with respect to the index-four sublattice generated by its edges; we denote this lattice by $\Lambda_{\mathcal{E}(P)}$. This restriction gives the polytope $P^{\prime}$ with vertices $\{(-9,-2,-4),(1,0,0),(3,2,0),(5,0,4)\}$. Similarly, restricting $2 P^{*}$ to the index-two sublattice $\Lambda_{\mathcal{E}\left(2 P^{*}\right)}$ yields a 1 -reflexive polytope $Q$ with vertices $\{(-1,1,1)$, $(-1,1,2),(-1,3,1),(3,-5,-4)\}$. Furthermore, $P^{*} \cong Q$.

Unfortunately, three-dimensional $l$-reflexive polytopes are not necessarily 1 -reflexive with respect to the sublattice generated by the edges. For consider the simplex $T$ associated with $\mathbb{P}(1,2,3,6)$, namely the convex hull of $\{(-2,-3,-6),(1,0,0),(0,1,0),(0,0,1)\}$. Let

$$
H:=\left(\begin{array}{lll}
4 & 0 & 1 \\
0 & 4 & 3 \\
0 & 0 & 1
\end{array}\right) \text {. }
$$

The resulting polytope $S:=T H$ with vertices $\{(-8,-12,-17),(4,0,1),(0,4,3),(0,0,1)\}$ is 2 -reflexive. If we restrict $S$ to the index-two sublattice $\Lambda_{\mathcal{E}(S)}$, the resulting simplex is not a reflexive polytope (it corresponds to fake weighted projective space $\mathbb{P}(1,2,3,6) /(\mathbb{Z} / 2 \times$ $\mathbb{Z} / 4))$.

\subsection{Classification algorithms in higher dimensions?}

Our classification algorithm in dimension two relies on the fact that for any $l$-reflexive polygon $P$ there exists a 1-reflexive polygon $Q$ such that $P$ is the image of an integer $2 \times 2$-matrix of determinant $l$ (Corollary 12). This motivates our main question:

Question: Is an $l$-reflexive polytope $P$ 1-reflexive with respect to the vertex lattice $\Lambda_{\mathcal{V}(P)} ?$

We do not know of a counterexample. However, even if this question has a positive answer, it does not immediately yield a general classification algorithm. Notice that the 2-reflexive polytope $S$ in Subsection 3.3 is the image under multiplication by a matrix of determinant $16>2$. This shows that it would be necessary to have an upper bound on the index of the vertex lattice of $l$-reflexive polytopes in dimension $n$, perhaps something analogous to Corollary 2.11 in [24]. This is not clear even for $l=1$.

\footnotetext{
${ }^{1}$ ID 547364 in the classification of toric canonical Fano threefolds [25]; see the online Graded Ring Database.
} 


\subsection{The edge lattice and the number 24}

Analogous to the "number 12"-property in dimension two, there is a well-known property of three-dimensional 1-reflexive polytopes refered to as the "number 24"-property:

Theorem 20 ([9, Theorem 4.3]). Let $P \subset N_{\mathbb{Q}}$ be a three-dimensional reflexive polytope. Then:

$$
\sum_{E \in \mathcal{E}(P)} \operatorname{Vol}(E) \cdot \operatorname{Vol}\left(E^{*}\right)=24
$$

where $E^{*}$ is the edge in $P^{*}$ corresponding to $E$, and $\operatorname{Vol}(E):=|E \cap N|-1$.

Let us again consider the first example of a 2-reflexive tetrahedron $P$ in Subsection 3.3. Since $P^{*} \cong Q$, we observe that a reformulation of Theorem 20 holds for the 2-reflexive polytope $P$ and its dual $2 P^{*}$, where we understand $E^{*}$ to mean the edge in $2 P^{*}$ corresponding to $E$.

On the other hand, in the second example of a 2-reflexive tetrahedron $S$ in Subsection 3.3, one computes that this generalised 24-property does not hold; the sum is 28 . These observations motivate the following conjecture:

Conjecture 21. If $P$ is a three-dimensional $l$-reflexive polytope such that $P$ restricted to $\Lambda_{\mathcal{E}(P)}$ is isomorphic to a 1-reflexive polytope $Q$, then $l P^{*}$ restricted to $\Lambda_{\mathcal{E}\left(l P^{*}\right)}$ is isomorphic to $Q^{*}$. In particular $P$ satisfies the 24 -property.

We checked this conjecture for all $l$-reflexive polytopes contained in the classification of all three-dimensional canonical Fano polytopes [25].

Remark 22. For a higher-dimensional version of the previous conjecture, we would expect to restrict to the sublattice generated by the codimension-two faces. Motivation for this comes from the main result in [18]: in dimension three or higher, the sublattice generated by all lattice points in a 1-reflexive polytope $P$ equals the sublattice generated by all lattice points in codimension-two faces of $P$.

\section{Acknowledgements}

The authors wish to thank the RG Lattice Polytopes at the Freie Universität Berlin, the Computational Algebra Group at the University of Sydney, and Imperial College London, for their hospitality and financial support. The authors are also grateful to an anonymous referee for some helpful suggestions. The first author is supported by EPSRC grant EP/I008128/1, the second author is supported by the US National Science Foundation (DMS 1203162). This work was supported in part by EPSRC Mathematics Platform grant EP/I019111/1.

\section{A Magma source code}

The following basic MAGMA code can be used to regenerate the classification of $l$-reflexive polygons. 
// Returns true iff $\mathrm{P}$ is 1-reflexive for some index 1. Also returns 1 . function is_l_reflexive(P)

if not IsFano(P) then return false, $;$; end if;

$1:=$ GorensteinIndex $(\mathrm{P})$;

if \&and[Denominator $(\mathrm{V})$ eq $1: \mathrm{V}$ in Vertices(Dual(P))] then return true, 1 ;

else

return false, _;

end if;

end function;

// Compute all non-isomorphic l-reflexive polygons generated by

// the Hermite normal forms with determinant 1.

procedure generate_polys(1, 〜polys)

if 1 eq 1 then

polys [1]:=[PolytopeReflexiveFanoDim2(id) : id in [1..16]]; return;

end if;

polys [l]: $=[]$;

Hs: $=[\operatorname{Matrix}(2,2,[1, i, 0,1])$ : i in $[1 . .1-1] \mid \operatorname{GCD}(1, i)$ eq 1];

for id in $[1 . .16]$ do

$\mathrm{P}:=$ PolytopeReflexiveFanoDim2(id);

for $\mathrm{H}$ in $\mathrm{Hs}$ do

$\mathrm{Q}:=\mathrm{P} * \mathrm{H}$;

bool, k:=is_l_reflexive(Q);

if bool and not \&or[IsIsomorphic $(Q, R): R$ in polys [k]] then Append ( polys $[k], Q)$;

end if;

end for;

end for;

end procedure;

// The main loop (runs from index 1 to 29, takes approx. 1 minute)

polys:=AssociativeArray (Integers ()) ;

for 1 in [1..29 by 2] do generate_polys(1, "polys); end for;

\section{References}

[1] Valery Alexeev and Viacheslav V. Nikulin. Del Pezzo and K3 surfaces, volume 15 of MSJ Memoirs. Mathematical Society of Japan, Tokyo, 2006.

[2] Christos A. Athanasiadis. Ehrhart polynomials, simplicial polytopes, magic squares and a conjecture of Stanley. J. Reine Angew. Math., 583:163-174, 2005. 
[3] Victor Batyrev and Benjamin Nill. Combinatorial aspects of mirror symmetry. In Integer points in polyhedra-geometry, number theory, representation theory, algebra, optimization, statistics, volume 452 of Contemp. Math., pages 35-66. Amer. Math. Soc., Providence, RI, 2008.

[4] Victor V. Batyrev. Dual polyhedra and mirror symmetry for Calabi-Yau hypersurfaces in toric varieties. J. Algebraic Geom., 3(3):493-535, 1994.

[5] Victor V. Batyrev and Lev A. Borisov. Mirror duality and string-theoretic Hodge numbers. Invent. Math., 126(1):183-203, 1996.

[6] Victor V. Batyrev and Lev A. Borisov. On Calabi-Yau complete intersections in toric varieties. In Higher-dimensional complex varieties (Trento, 1994), pages 39-65. de Gruyter, Berlin, 1996.

[7] Victor V. Batyrev and Lev A. Borisov. Dual cones and mirror symmetry for generalized Calabi-Yau manifolds. In Mirror symmetry, II, volume 1 of AMS/IP Stud. Adv. Math., pages 71-86. Amer. Math. Soc., Providence, RI, 1997.

[8] M. Beck, J. A. De Loera, M. Develin, J. Pfeifle, and R. P. Stanley. Coefficients and roots of Ehrhart polynomials. In Integer points in polyhedra-geometry, number theory, algebra, optimization, volume 374 of Contemp. Math., pages 15-36. Amer. Math. Soc., Providence, RI, 2005.

[9] Matthias Beck, Beifang Chen, Lenny Fukshansky, Christian Haase, Allen Knutson, Bruce Reznick, Sinai Robins, and Achill Schürmann. Problems from the Cottonwood Room. In Integer points in polyhedra - geometry, number theory, algebra, optimization, volume 374 of Contemp. Math., pages 179-191. Amer. Math. Soc., Providence, RI, 2005.

[10] Matthias Beck and Sinai Robins. Computing the continuous discretely. Undergraduate Texts in Mathematics. Springer, New York, 2007. Integer-point enumeration in polyhedra.

[11] Christian Bey, Martin Henk, and Jörg M. Wills. Notes on the roots of Ehrhart polynomials. Discrete Comput. Geom., 38(1):81-98, 2007.

[12] Winfried Bruns and Tim Römer. $h$-vectors of Gorenstein polytopes. J. Combin. Theory Ser. A, 114(1):65-76, 2007.

[13] Cinzia Casagrande. The number of vertices of a Fano polytope. Ann. Inst. Fourier (Grenoble), 56(1):121-130, 2006.

[14] Heinke Conrads. Weighted projective spaces and reflexive simplices. Manuscripta Math., 107(2):215-227, 2002.

[15] Dimitrios I. Dais. Classification of toric log del Pezzo surfaces having Picard number 1 and index $\leqslant 3$. Results Math., 54(3-4):219-252, 2009.

[16] Matthew H. J. Fiset and Alexander M. Kasprzyk. A note on palindromic $\delta$-vectors for certain rational polytopes. Electron. J. Combin., 15(1):Note 18, 4, 2008. 
[17] William Fulton. Introduction to toric varieties, volume 131 of Ann. of Math. Stud. Princeton University Press, Princeton, NJ, 1993. The William H. Roever Lectures in Geometry.

[18] Christian Haase and Benjamin Nill. Lattices generated by skeletons of reflexive polytopes. J. Combin. Theory Ser. A, 115(2):340-344, 2008.

[19] Christian Haase and Josef Schicho. Lattice polygons and the number $2 i+7$. Amer. Math. Monthly, 116(2):151-165, 2009.

[20] Gábor Hegedüs and Alexander Kasprzyk. Roots of Ehrhart polynomials of smooth Fano polytopes. Discrete and Computational Geometry, 46(3):488-499, 2011.

[21] Takayuki Hibi. Dual polytopes of rational convex polytopes. Combinatorica, 12(2):237-240, 1992.

[22] Takayuki Hibi, Akihiro Higashitani, and Hidefumi Ohsugi. Roots of Ehrhart polynomials of Gorenstein Fano polytopes. Proc. Amer. Math. Soc., 139(10):3727-3734, 2011.

[23] Lutz Hille and Harald Skarke. Reflexive polytopes in dimension 2 and certain relations in $S L_{2}(\mathbb{Z})$. J. Algebra Appl., 1(2):159-173, 2002.

[24] Alexander M. Kasprzyk. Bounds on fake weighted projective space. Kodai Math. J., 32:197-208, 2009.

[25] Alexander M. Kasprzyk. Canonical toric Fano threefolds. Canad. J. Math., 62(6):1293-1309, 2010.

[26] Alexander M. Kasprzyk, Maximilian Kreuzer, and Benjamin Nill. On the combinatorial classification of toric log del pezzo surfaces. LMS Journal of Computation and Mathematics, 13:33-46, 2010.

[27] Maximilian Kreuzer and Harald Skarke. On the classification of reflexive polyhedra. Comm. Math. Phys., 185(2):495-508, 1997.

[28] Maximilian Kreuzer and Harald Skarke. Complete classification of reflexive polyhedra in four dimensions. Adv. Theor. Math. Phys., 4(6):1209-1230, 2000.

[29] Jeffrey C. Lagarias and Günter M. Ziegler. Bounds for lattice polytopes containing a fixed number of interior points in a sublattice. Canad. J. Math., 43(5):1022-1035, 1991.

[30] Noboru Nakayama. Classification of log del Pezzo surfaces of index two. J. Math. Sci. Univ. Tokyo, 14(3):293-498, 2007.

[31] Benjamin Nill. Gorenstein toric Fano varieties. Manuscripta Math., 116(2):183-210, 2005.

[32] Mikkel Øbro. An algorithm for the classification of smooth Fano polytopes. April 2007. arXiv:0704.0049v1, classifications available from http://grdb.lboro.ac.uk/.

[33] Bjorn Poonen and Fernando Rodriguez-Villegas. Lattice polygons and the number 12. Amer. Math. Monthly, 107(3):238-250, 2000. 